\title{
Construction of Fusion Frame Systems in Finite Dimensional Hilbert Spaces
}

\author{
Jinsong Leng and Tingzhu Huang \\ School of Mathematical Sciences, University of Electronic Science and Technology of China, Chengdu 610054, China \\ Correspondence should be addressed to Jinsong Leng; jslengjs@gmail.com
}

Received 26 November 2013; Accepted 25 January 2014; Published 12 March 2014

Academic Editor: Carlo Cattani

Copyright (C) 2014 J. Leng and T. Huang. This is an open access article distributed under the Creative Commons Attribution License, which permits unrestricted use, distribution, and reproduction in any medium, provided the original work is properly cited.

\begin{abstract}
We first investigate the construction of a fusion frame system in a finite-dimensional Hilbert space $\mathbb{F}^{n}$ when its fusion frame operator matrix is given and provides a corresponding algorithm. The matrix representations of its local frame operators and inverse frame operators are naturally obtained. We then study the related properties of the constructed fusion frame systems. Finally, we implement the construction of fusion frame systems which behave optimally for erasures in some special sense in signal transmission.
\end{abstract}

\section{Introduction}

The theory of frames has gradually become an attractive research area in the past twenty years. A prominent feature of frames is redundancy which has two advantages: it makes the construction of various frames more flexible and it provides stability and robustness of signal in transmission. This leads to the rapid development of theory and applications of frames in past twenty years. We refer to [1-3] and the references therein for more details about the frame theory and its new achievements. In applications, we only mention some areas here such as signal and image processing [4], quantization [5], capacity of transmission channel $[1,2,6]$, coding theory [7-12], and data transmission technology [13].

But in some modern applications, the data which need to be handled are so large that the processing procedures cannot be implemented effectively by using a single frame. Fusion frames are naturally suitable tools for dealing with this problem. One can see the systemic introduction of theory of fusion frames in $[14,15]$. In recent years, many excellent results about the theory and applications of fusion frames have been achieved at an amazing speed [15-20]. In fact, fusion frames are generalization of conventional frames and go beyond them. The procedure of using fusion frame systems to handle information can be described as follows. A large number of data can be assigned to a set of small spaces and processed in these subsystems, finally all the information are fused together at a center. Fusion frames have been applied to various fields where distributed or parallel processing is required. For instance, in a coding transmission process, the encoded and quantized data must be put in numbers of packets. When one or more packets are scrambled, lost, or delayed, fusion frames can enhance the robustness to the packet erasures. Furthermore, we can see the successful applications of fusion frames in sensors network [21], transmission coding [22-25], and so forth.

However, some problems about fusion frame systems are open. Many excellent results about conventional frames have been obtained and applied successfully, but how to generalize them to fusion frames? Even in mathematics application, the relation between the theory of fusion frames and the interesting fields studied in [26-28] is worth further researching. It is an appealing subject due to the complexity of the structure of fusion frames compared with conventional frames. In this paper, we focus on the matrix representations of fusion frame operators of fusion frame systems and the construction of fusion frame systems if their fusion frame operator matrices are provided. To this end, we first study the correspondence between frames of a subspace $W$ with dimension $l$ of an $n$-dimensional Hilbert space $\mathscr{H}$ with frames of Hilbert space $\mathbb{F}^{l}$, where $l \leq n$. We obtain the matrix representations of the local inverse frame operators and the 
fusion frame operator of a given fusion frame system by using the correspondence. Based on these matrix representations, the concrete algorithm for constructing a dual fusion frame system is provided. Then we investigate the construction of fusion frame systems, which fusion frame operators are given. It is essential for constructing fusion frame systems to get their local frames. We show that the constructed local frame of a subspace with dimension $l$ can inherit some properties from the corresponding frame of Hilbert space $\mathbb{F}^{l}$ such as Parseval and harmony. Finally, we give a method for construction of the optimal fusion frame systems for one local frame vector erasure.

We organize the structure of this paper as follows. In Section 2, we introduce and recall some notations, conceptions, and some basic theory about frames and fusion frame systems. Then we recall the method to obtain the matrix representation of the fusion frame operator of a given fusion frame system in a finite-dimensional Hilbert space $\mathbb{F}^{n}$. In Section 3, we study the construction of frames of an $l$ dimensional subspace $W$ of $\mathbb{F}^{n}$ by using the corresponding frames of $\mathbb{F}^{l}$, where $l \leq n$. We then present an algorithm for constructing a fusion frame system when its fusion frame operator is given. Moreover, we get the matrix representations of its local frame operators and inverse frame operators and research the related characteristics of the constructed fusion frame systems. The optimal fusion frame systems under erasures in some particular sense can be obtained by using our method. An example is given to show the effectiveness of our construction in imagine coding.

\section{Preliminaries}

We refer to $[1-3,15,25]$ for the details of the basic notations, concepts, and results about frames and fusion frame systems. We will adopt the same notations as [25] throughout this paper. We recall the main concepts and results about the construction of the matrix representation of the fusion frame operator of a given fusion frame system in this section.

Let $\mathscr{W}=\left\{\left(W_{i}, v_{i}\right)\right\}_{i \in I}$ be a fusion frame for $\mathscr{H}$. The analysis operator $\Theta_{\mathscr{W}}$ is defined by

$$
\Theta_{\mathscr{W}}: \mathscr{H} \longrightarrow\left(\sum_{i \in I} \oplus W_{i}\right)_{\ell_{2}} \text { with } \Theta_{\mathscr{W}}(f)=\left\{v_{i} P_{W_{i}}(f)\right\}_{i \in I},
$$

where

$$
\left(\sum_{i \in I} \oplus W_{i}\right)_{\ell_{2}}=\left\{\left\{f_{i}\right\}_{i \in I} \mid f_{i} \in W_{i},\left\{\left\|f_{i}\right\|\right\}_{i \in I} \in \ell^{2}(I)\right\}
$$

is called the representation space. The synthesis operator $\Theta_{\mathscr{W}}^{*}$ (the adjoint operator of $\Theta_{\mathscr{W}}$ ) can be defined by

$$
\begin{gathered}
\Theta_{\mathscr{W}}^{*}:\left(\sum_{i \in I} \oplus W_{i}\right)_{\ell_{2}} \longrightarrow \mathscr{H} \quad \text { with } \Theta_{\mathscr{W}}^{*}(f)=\sum_{i \in I} v_{i} f_{i}, \\
f=\left\{f_{i}\right\}_{i \in I} \in\left(\sum_{i \in I} \oplus W_{i}\right)_{\ell_{2}} .
\end{gathered}
$$

The fusion frame operator $S_{\mathscr{W}}$ for $\mathscr{W}$ is defined by

$$
S_{\mathscr{W}}(f)=\Theta_{\mathscr{W}}^{*} \Theta_{\mathscr{W}}(f)=\sum_{i \in I} v_{i}^{2} P_{W_{i}}(f)
$$

The following result shows how to obtain the global dual frame from the local dual frames.

Proposition 1 (c.f. [15], Proposition 4.3). Let $\left\{\left(W_{i}, v_{i}\right.\right.$, $\left.\left.\left\{f_{i j}\right\}_{j \in J_{i}}\right)\right\}_{i \in I}$ be a fusion frame system for $\mathscr{H}$ with associated fusion frame operator $S_{\mathscr{W}}$, common local frame bounds, and local dual frames $\left\{\tilde{f}_{i j}\right\}_{j \in J_{i}}, i \in I$. Then $\left\{v_{i} S_{\mathscr{W}}^{-1}\left(\tilde{f}_{i j}\right)\right\}_{j \in J_{i}, i \in I}$ is a dual frame for the frame $\left\{v_{i} f_{i j}\right\}_{j \in J_{i}, i \in I}$.

Because we only consider finite-dimensional Hilbert spaces, $I$ will denote the identity operator (matrix) exclusively in the rest of the paper.

Let $\left\{\left(W_{i}, v_{i},\left\{f_{i j}\right\}_{j=1}^{k_{i}}\right)\right\}_{i=1}^{m}$ be a fusion frame system for $\mathbb{F}^{n}$; then the analysis operator of the local frame of $W_{i}$ is a $k_{i} \times n$ matrix $\Theta_{F_{i}}$ with $f_{i j}^{*}$ as its $j$ th row and the $n \times k_{i}$ matrix $\Theta_{F_{i}}^{*}$ is its synthesis operator. Furthermore, the $i$ th local frame operator is an $n \times n$ matrix $S_{F_{i}}=\Theta_{F_{i}}^{*} \Theta_{F_{i}}$.

Notation. For the purpose of coding of any $f \in \mathbb{F}^{n}, \Theta_{F_{i}}$ always denote the analysis operator of the system $\left\{f_{i j}\right\}_{j=1}^{k_{i}}$ in $\mathbb{F}^{n}$ throughout this paper. Hence it is a $k_{i} \times n$ matrix, not a $k_{i} \times\left(\operatorname{dim} W_{i}\right)$ matrix.

The following definition is given by [25].

Definition 2. Let $W$ be an $l$-dimensional subspace of $\mathbb{F}^{n}$ with a local frame $F=\left\{f_{i}\right\}_{i=1}^{k}$, where $l \leq n$. $S_{F}$ is the local frame operator of $F$. If there exists an operator $A$ such that $f=S_{F} A(f)=A S_{F}(f)$ holds for all $f \in W$, we call $A$ the inverse of $S_{F}$ in $W$ and denote it by $S_{F}^{-1}$.

For any $f \in \mathbb{F}^{n}, \Theta_{F_{i}} f$ is its encoding version in subspace $W_{i}$. For obtaining $P_{W_{i}}(f)=\sum_{j=1}^{k_{i}}\left\langle f, f_{i j}\right\rangle \widetilde{f}_{i j}=$ $\sum_{j=1}^{k_{i}}\left\langle f, f_{i j}\right\rangle S_{F_{i}}^{-1} f_{i j}=\Theta_{\widetilde{F}_{i}}^{*} \Theta_{F_{i}}(f)$, the following lemma is given to calculate the matrix representation of $S_{F_{i}}^{-1}$ and the $i$ th local dual frame $\left\{\tilde{f}_{i j}\right\}_{j=1}^{k_{i}}$.

Lemma 3 (c.f. [25], Lemma 11). Let $W$ be an l-dimensional subspace of $\mathbb{F}^{n}$ with an orthonormal basis $\left\{e_{i}\right\}_{i=1}^{l}$ and a frame $F=\left\{f_{i}\right\}_{i=1}^{k}$ with frame bounds $A, B$, wherel $\leq n$. Define $L$ to be an $l \times n$ matrix with the vector $e_{i}^{*}$ as its ith row for $i=1,2, \ldots, l$, where $e_{i}^{*}$ is the conjugate-transpose of $e_{i}$. The sequence $G=$ $\left\{g_{i}\right\}_{i=1}^{k}$ is given by $g_{i}=L f_{i}$ for $i=1,2, \ldots, k$. Then $\left\{g_{i}\right\}_{i=1}^{k}$ is a frame of $\mathbb{F}^{l}$ with the same frame bounds as $F$. In particular, if $F$ is a tight (or Parseval) frame, also is $G$.

By applying this lemma, we can obtain a method to compute the matrix representation of the inverse frame operator of a subspace endowed with an orthonormal basis in the following theorem.

Theorem 4 (c.f. [25], Theorem 12). Let W be an l-dimensional subspace of $\mathbb{F}^{n}$ with an orthonormal basis $\left\{e_{i}\right\}_{i=1}^{l}$ and a frame 
$F=\left\{f_{i}\right\}_{i=1}^{k}$, where $l \leq n$. L is defined as the above lemma. $S_{F}$ is the frame operator of $F$. Then

$$
S_{F}^{-1}=L^{*}\left(L S_{F} L^{*}\right)^{-1} L
$$

is the inverse of $S_{F}$ in $W$. Moreover, the orthogonal projection $P_{W}$ from $F^{n}$ onto $W$ is $P_{W}=S_{F}^{-1} S_{F}=S_{F} S_{F}^{-1}=L^{*} L$.

For a given fusion frame system $\left\{\left(W_{i}, v_{i}, F_{i}=\left\{f_{i j}\right\}_{j=1}^{k_{i}}\right)\right\}_{i=1}^{m}$, we can calculate the orthonormal basis of its each subspace by finding the maximally linear independent subset of $F_{i}$ and taking the Gram-Schmidt process on it. Then by using the above theorem, we derive the matrix representations of all local inverse frame operators and orthogonal projections onto the subspaces $\left\{W_{i}\right\}_{i=1}^{m}$. Furthermore, we can compute the matrix representation of the fusion frame operator by applying the formula provided by the following proposition.

Proposition 5 (c.f. [25], Proposition 13). Let $\left\{\left(W_{i}, v_{i}, F_{i}=\right.\right.$ $\left.\left.\left\{f_{i j}\right\}_{j=1}^{k_{i}}\right)\right\}_{i=1}^{m}$ be a fusion frame system for $\mathbb{F}^{n}$, and let $\widetilde{F}_{i}=$ $\left\{\tilde{f}_{i j}\right\}_{j \in J_{i}}, i \in I$, be the local dual frames given by $\tilde{f}_{i j}=S_{F_{i}}^{-1} f_{i j}$ for all $j=1,2, \ldots, k_{i}, i=1,2, \ldots, m$. Then the matrix representation of the fusion frame operator is given by

$$
\begin{aligned}
S_{\mathscr{W}} & =\sum_{i=1}^{m} v_{i}^{2} \Theta_{\widetilde{F}_{i}}^{*} \Theta_{F_{i}}=\sum_{i=1}^{m} v_{i}^{2} \Theta_{F_{i}}^{*} \Theta_{\widetilde{F}_{i}} \\
& =\sum_{i=1}^{m} v_{i}^{2} S_{F_{i}}^{-1} S_{F_{i}}=\sum_{i=1}^{m} v_{i}^{2} S_{F_{i}} S_{F_{i}}^{-1},
\end{aligned}
$$

where $\Theta_{F_{i}}$ and $\Theta_{\widetilde{F}_{i}}$ are the analysis operators of $F_{i}$ and $\widetilde{F}_{i}$, respectively, and $S_{F_{i}}$ is the frame operator of $F_{i}$ for each $i \in I$.

Given a fusion frame system of a finite-dimensional Hilbert space $\mathbb{F}^{n}$, the matrix representation of its fusion frame operator as well as its one dual fusion frame system can be obtained by using the above two results. The concrete algorithm is presented in [25].

\section{Construction of Fusion Frame Systems}

In this section, we research the construction of a fusion frame system with a given positive invertible matrix $S$ as its fusion frame operator. The constructing approach should include two stages. First, construct the orthogonal projections of all subspaces as well as their weights if the fusion frame operator is given. Secondly, construct the local frames of all subspaces with the desired properties. By using this method, we can derive the optimal fusion frame systems for erasures in some special sense.

3.1. Construction of Fusion Frame Systems. We first recall our previous work in [19] on the construction of fusion frames which fusion frame operators are provided. In practise, the local frames of a fusion frame system are served as coder in their respective subspaces. The main distribution of this subsection is the derivation of the local frames with the expected characteristics which can be implemented by constructing frames of $\mathbb{F}^{l_{i}}$ with the same dimension as subspace $W_{i}$ for each $i \in\{1,2, \ldots, m\}$. And then, we get the construction of fusion frame systems combined with the previous work.

Notations and Assumptions. We set up some notations that will be used throughout this subsection. Let $S$ be a positive $n \times n$ matrix with eigenvalues $\left\{\lambda_{i}\right\}_{i=1}^{n}$ where $\lambda_{i}>0$ for all $1 \leq$ $i \leq n$ and let $e_{i}, i=1,2, \ldots, n$ be the orthonormal eigenvectors of $S$ corresponding to the eigenvalues $\lambda_{i}$, respectively, which form an orthonormal basis of $\mathbb{F}^{n}$. Let $m$ be a positive integer, and $L_{i}=\left(e_{i 1}, e_{i 2}, \ldots, e_{i l_{i}}\right)^{*}$; that is, the matrix $L_{i}$ is constituted by $e_{i j}^{*}, j=1,2, \ldots, l_{i}$ as its rows for $i=1,2, \ldots, m$, where $e_{i j} \in\left\{e_{i}\right\}_{i=1}^{n}$ and $e_{i j_{1}} \neq e_{i j_{2}}$ when $j_{1} \neq j_{2}$. Assume that the matrix $\left[L_{1}^{*}, L_{2}^{*}, \ldots, L_{m}^{*}\right]$ has rank $n$; that is, the rows of all $L_{i}, i=1,2, \ldots, m$ can span the space $\mathbb{F}^{n}$. Set $\Lambda_{i}=\{j \mid$ $e_{i}^{*}$ is one of the rows of $\left.L_{j}\right\}$ for $i=1,2, \ldots, n$.

The following theorem provides the method of orthogonal projection decomposition of a given positive matrix.

Theorem 6 (c.f. [19], Theorem 3.2). Let the notations and assumptions be as described in the previous setup. Let the positive numbers $\left\{v_{i}\right\}_{i=1}^{m}$ satisfy the following condition:

$$
\sum_{j \in \Lambda_{i}} v_{j}^{2}=\lambda_{i}
$$

for all $i=1,2, \ldots, m$; then the positive matrix $S$ has the following decomposition:

$$
S=\sum_{i=1}^{m} v_{i}^{2} P_{i}
$$

where $P_{i}=L_{i}^{*} L_{i}, i=1,2, \ldots, m$ are orthogonal projection matrices on $W_{i}=\operatorname{span}\left\{e_{i_{j}}\right\}_{j=1}^{l_{i}}$.

The following proposition provides a method for the construction of a fusion frame as well as orthonormal projections on its subspaces with a given fusion frame operator.

Proposition 7 (c.f. [19], Proposition 3.4). Let the notations and assumptions be as described in the previous setup, and the positive numbers $\left\{v_{i}\right\}_{i=1}^{m}$ satisfy equation (7). Then $\left\{\left(W_{i}, v_{i}\right)\right\}_{i=1}^{m}$ is a fusion frame for $\mathbb{F}^{n}$ with frame operator $S_{\mathscr{W}}=S$, where $W_{i}=\operatorname{span}\left\{e_{i_{j}}\right\}_{j=1}^{l_{i}}$. In the case that $S=A I$, we have that if $\left\{v_{i}\right\}_{i=1}^{m}$ satisfies the following condition:

$$
\sum_{j \in \Lambda_{i}} v_{j}^{2}=A
$$

for all $i=1,2, \ldots, m$, where $A>0$ is a positive real number; then $\left\{\left(W_{i}, v_{i}\right)\right\}_{i=1}^{m}$ is an A-tight fusion frame for $\mathbb{F}^{n}$. In particular, if $A=1$, then it is a Parseval fusion frame.

Then we will focus on the construction of local frames of the fusion frames derived by the above proposition. It is an important step for constructing fusion frame systems. We first show the following theorem which is the converse of Lemma 3. 
Theorem 8. Let $W$ be an l-dimensional subspace of $\mathbb{F}^{n}$ with an orthonormal basis $\left\{e_{i}\right\}_{i=1}^{l}$, and let $G=\left\{g_{i}\right\}_{i=1}^{k}$ be a frame of $\mathbb{F}^{l}$ with frame bounds $A, B$, where $l \leq n$. L is defined as Lemma 3. The sequence $F=\left\{f_{i}\right\}_{i=1}^{k}$ is given by $f_{i}=L^{*} g_{i}$ for $i=1,2, \ldots, k$. Then $F$ is a frame of $W$ with the same frame bounds as $G$. In particular, if $G$ is a tight (or Parseval) frame, also is $F$.

Proof. For any $f \in W$, we have $L f=$ $\left(\left\langle f, e_{1}\right\rangle,\left\langle f, e_{2}\right\rangle, \ldots,\left\langle f, e_{l}\right\rangle\right)^{T} \in \mathbb{F}^{l}$ and $\|L f\|^{2}=$ $\sum_{i=1}^{l}\left|\left\langle f, e_{i}\right\rangle\right|^{2}=\|f\|^{2}$. Therefore,

$$
\begin{aligned}
A\|f\|^{2} & =A\|L f\|^{2} \leq \sum_{i=1}^{k}\left|\left\langle L f, g_{i}\right\rangle\right|^{2}=\sum_{i=1}^{k}\left|\left\langle f, L^{*} g_{i}\right\rangle\right|^{2} \\
& =\sum_{i=1}^{k}\left|\left\langle f, f_{i}\right\rangle\right|^{2} \leq B\|L f\|^{2}=B\|f\|^{2}
\end{aligned}
$$

as required. The particular assertion is obvious.

The following proposition gives the matrix representations of the local frame operator and inverse frame operator of a subspace $W$ of $\mathbb{F}^{n}$ derived by the above theorem.

Proposition 9. Let $W$ be an l-dimensional subspace of $\mathbb{F}^{n}$ with an orthonormal basis $\left\{e_{i}\right\}_{i=1}^{l}$, and let $G=\left\{g_{i}\right\}_{i=1}^{k}$ be a frame of $\mathbb{F}^{l}$ with frame bounds $A, B$, where $l \leq n$. L and $F=\left\{f_{i}\right\}_{i=1}^{k}$ are defined as the above theorem. $S_{G}$ is the frame operator of $G$. Then the frame operator of $F$ and its inverse are given by

$$
\begin{gathered}
S_{F}=L^{*} S_{G} L, \\
S_{F}^{-1}=L^{*} S_{G}^{-1} L .
\end{gathered}
$$

Moreover, the orthogonal projection $P_{W}$ from $F^{n}$ onto $W$ is $P_{W}=S_{F}^{-1} S_{F}=S_{F} S_{F}^{-1}=L^{*} L$.

Proof. Let $\Theta_{G}$ and $\Theta_{G}^{*}$ be the analysis operator and synthesis operator of $G$, respectively; then $\Theta_{F}^{*}=$ $\left(L^{*} g_{1}, L^{*} g_{2}, \ldots, L^{*} g_{k}\right)=L^{*} \Theta_{G}^{*}$ is the synthesis operator of $F$. Hence, the frame operator of $F$ is $S_{F}=\Theta_{F}^{*} \Theta_{F}=$ $L^{*} \Theta_{G}^{*} \Theta_{G} L=L^{*} S_{G} L$.

Since $S_{G}$ is invertible, we will show that $S_{F}^{-1}=L^{*} S_{G}^{-1} L$ is inverse of $S_{F}$. Note that $L L^{*}=I$ and $L^{*} L f=f$ for any $f \in W$. Hence, we have

$$
\begin{aligned}
& S_{F}^{-1} S_{F} f=L^{*} S_{G}^{-1} L L^{*} S_{G} L f=L^{*} L f=f \\
& S_{F} S_{F}^{-1} f=L^{*} S_{G} L L^{*} S_{G}^{-1} L f=L^{*} L f=f,
\end{aligned}
$$

as claimed. The proof of the moreover part is the same as that of Theorem 12 of [25].

The following two propositions show that the constructed frame of $W$ inherits some features of the corresponding frame of $\mathbb{F}^{l}$.

Proposition 10. Let $W$ be an l-dimensional subspace of $\mathbb{F}^{n}$ with an orthonormal basis $\left\{e_{i}\right\}_{i=1}^{l}$, and let $G=\left\{g_{i}\right\}_{i=1}^{k}$ be a frame of $\mathbb{F}^{l}$ with frame bounds $A, B$, where $l \leq n$. L and $F=\left\{f_{i}\right\}_{i=1}^{k}$ are defined as Theorem 8. If $\widetilde{G}=\left\{\widetilde{g}_{i}\right\}_{i=1}^{k}$ is a dual frame of $G$, then $\widetilde{F}=\left\{L^{*} \widetilde{g}_{i}\right\}_{i=1}^{k}$ is a dual frame of $F$. Furthermore, if $\widetilde{G}$ is the canonical one, so is $\widetilde{F}$.

Proof. For any $f \in W$, we have

$$
\begin{aligned}
\sum_{i=1}^{k}\left\langle f, L^{*} g_{i}\right\rangle L^{*} \tilde{g}_{i} & =L^{*} \sum_{i=1}^{k}\left\langle L f, g_{i}\right\rangle \tilde{g}_{i}=\sum_{i=1}^{k}\left\langle f, L^{*} \tilde{g}_{i}\right\rangle L^{*} g_{i} \\
& =L^{*} \sum_{i=1}^{k}\left\langle L f, \tilde{g}_{i}\right\rangle g_{i}=L^{*} L f=f .
\end{aligned}
$$

Hence, $\widetilde{F}$ is a dual frame of $F$. If $\widetilde{g}_{i}=S_{G}^{-1} g_{i}$, then $\widetilde{f}_{i}=L^{*} \widetilde{g}_{i}=$ $L^{*} S_{G}^{-1} L L^{*} g_{i}=S_{F}^{-1} f_{i}$ for $i=1,2, \ldots, k$, which implies that $\widetilde{F}$ is the canonical dual frame of $F$.

Proposition 11. Let $W$ be an l-dimensional subspace of $\mathbb{F}^{n}$ with an orthonormal basis $\left\{e_{i}\right\}_{i=1}^{l}$, and let $G=\left\{g_{i}\right\}_{i=0}^{k-1}$ be a harmonic frame of $\mathbb{F}^{l}$, where $l \leq n$. L and $F=\left\{f_{i}\right\}_{i=0}^{k-1}$ are defined as Theorem 8 . Then $F$ is a harmonic frame of $W$.

Proof. Since $G$ is a harmonic frame of $\mathbb{F}^{l}$, there exists a unitary $U$ on $\mathbb{F}^{l}$ such that $U^{k}=I, U^{i} \neq I$ for $1 \leq i \leq k-1$, and $g_{i}=U^{i} g_{0}$ for $0 \leq i \leq k-1$. Let $L^{*} U L=V$. For any $f \in W$, we have

$$
\begin{aligned}
V V^{*} f & =L^{*} U L L^{*} U^{*} L f=V^{*} V f=L^{*} U^{*} L L^{*} U L f \\
& =L^{*} L f=f .
\end{aligned}
$$

Therefore, $V$ is a unitary on $W$. It is obvious that $V^{i}=L^{*} U^{i} L$. So we have $V^{k}=L^{*} L, V^{i} \neq L^{*} L$ for $1 \leq i \leq k-1$, and $f_{i}=$ $L^{*} g_{i}=L^{*} U^{i} g_{0}=L^{*} U^{i} L L^{*} g_{0}=V^{i} f_{0}$, which implies that $F$ is a harmonic frame.

Summarizing the related results of this subsection, we can obtain the algorithm for constructing a required fusion frame system with a given fusion frame operator $S$ as follows.

Step 1. Compute the eigenvalues $\left\{\lambda_{i}\right\}_{i=1}^{n}$ and their corresponding independent eigenvectors $\left\{h_{i}\right\}_{i=1}^{n}$ of $S$.

Step 2. Take the Gram-Schmidt process on $\left\{h_{i}\right\}_{i=1}^{n}$ to get an orthonormal basis $\left\{e_{i}\right\}_{i=1}^{n}$ for $\mathbb{F}^{n}$.

Step 3. According to the requirement, construct the matrix $L_{i}$ constituted by this basis as follows:

$$
L_{i}=\left[\begin{array}{c}
\longleftarrow e_{i 1}^{*} \longrightarrow \\
\longleftarrow e_{i 2}^{*} \longrightarrow \\
\vdots \\
\longleftarrow e_{i l_{i}}^{*} \longrightarrow
\end{array}\right],
$$

for $i=1,2, \ldots, m$, where $e_{i j} \in\left\{e_{i}\right\}_{i=1}^{n}$, and $e_{i j_{1}} \neq e_{i j_{2}}$, when $j_{1} \neq j_{2}$. 
Step 4. Resolve (7) to derive the sequence of weights $\left\{v_{i}\right\}_{i=1}^{n}$. Set $W_{i}=\operatorname{span}\left\{e_{i_{j}}\right\}_{j=1}^{l_{i}}$. Use formula (8) to decompose $S$ and get the orthogonal projections $P_{W_{i}}$, for $i=1,2, \ldots, m$. Then we obtain a required fusion frame $\left\{\left(W_{i}, v_{i}\right)\right\}_{i=1}^{m}$.

Step 5. Construct the frames $G_{i}=\left\{g_{i j}\right\}_{j=1}^{k_{i}}$ in $\mathbb{F}^{l_{i}}$ with requirement properties for $i=1,2, \ldots, m$.

Step 6. Apply Theorem 8 to compute the local frames $F_{i}=$ $\left\{f_{i j}\right\}_{j=1}^{k_{i}}$ for $i=1,2, \ldots, m$. Then we derive a required fusion frame system $\left\{\left(W_{i}, v_{i},\left\{f_{i j}\right\}_{j=1}^{k_{i}}\right)\right\}_{i=1}^{m}$.

3.2. Construction of Optimal Fusion Frame Systems for Erasures. We apply our construction method to obtain optimal Parseval fusion frame systems for the packet erasure problem in some special sense in this subsection. Bodmann initiated in [22] the investigation about the optimality of $(m, k, n)$ protocols that are used to the packet erasure problem. Let $\left\{B_{j}\right\}_{j=1}^{m}$ be a family of coordinate operators $B_{j}: \mathscr{H} \rightarrow \mathscr{K}$ into a finite-dimensional Hilbert space $\mathscr{K}$ of maximal rank $k$ that provide a resolution of the identity $I=\sum_{j=1}^{m} B_{j}^{*} B_{j}$ for the Hilbert space $\mathscr{H}=\mathbb{F}^{n}$, where $m, k, n$ are positive integers satisfying $n<m k$; then the analysis operator $\Theta$ of such a family $\left\{B_{j}\right\}$ is called a $(m, k, n)$-protocol. The optimality of $(m, k, n)$-protocols requires to get weighted projective resolutions of the identity operator: $I=\sum_{j=1}^{m} B_{j}^{*} B_{j}=$ $\sum_{j=1}^{m} v_{i} P_{j}$, where $v_{j}>0$, and $P_{j}$ is a projection on some Hilbert space with rank- $k$ for $j=1,2, \ldots, m$. This can be also phrased by Parseval fusion frames (Theorem 3.6 of [18]). Furthermore, optimal Parseval fusion frame systems for one local frame vector erasure have been depicted in Theorem 4.3 of [18]). We point out that a special type of Parseval fusion frames that are optimal for the one packet erasure problem can be easily constructed by using Proposition 7. Moreover, Parseval fusion frame systems that are optimal for the one local frame vector erasure problem described by [18] can be easily constructed by using Theorem 8 . Let us recall the description of the optimal Parseval fusion frames for the one packet erasure problem.

Definition 12. Let $\mathscr{W}=\left\{\left(W_{i}, v_{i}\right)\right\}_{i=1}^{m}$ be a Parseval fusion frame for an $n$-dimension Hilbert space $\mathbb{F}^{n}$ with analysis operator $\Theta_{\mathscr{W}}$. Define the operator $D_{j}:\left(\sum_{i=1}^{m} \bigoplus W_{i}\right)_{\ell_{2}} \rightarrow$ $\left(\sum_{i=1}^{m} \oplus W_{i}\right)_{\ell_{2}}$ by $\left\{D_{j}(g)\right\}_{i}=\delta_{j i} g_{i}$ for all $i=1,2, \ldots, m$, where $g=\left\{g_{i}\right\}_{i=1}^{m} \in\left(\sum_{i=1}^{m} \bigoplus W_{i}\right)_{\ell_{2}}$. For any $f \in \mathscr{H}$, we call $D_{j} \Theta_{\mathscr{W}} f$ the $j$ th coding packet for $j=1,2, \ldots, m$. The one packet erasure reconstruction error $e_{1}(\mathscr{W})$ of $\mathscr{W}$ is defined by

$$
e_{1}(\mathscr{W})=\max \left\{\left\|\Theta_{\mathscr{W}}^{*} D_{i} \Theta_{\mathscr{W}}\right\|: 1 \leq i \leq m\right\} .
$$

In practise, a signal (vector) $f \in \mathscr{H}$ is encoded as $\Theta_{\mathscr{W}} f$ including $m$ coding packets and decoded (reconstructed) as $\Theta_{\mathscr{W}}^{*} \Theta_{\mathscr{W}} f$ by using a Parseval fusion frame $\mathscr{W}$. If one packet is lost in the transmission process, then $e_{1}(\mathscr{W})$ depict the reconstruction error in the worst case. The optimal Parseval fusion frame can be used to implement the optimal coding in this special sense in applications $[18,22]$. The following theorem describe the optimal Parseval fusion frames with a prescribed number of subspaces and prescribed dimensions of the subspaces under one subspace (packet) erasure.

Theorem 13 (c.f. [18], Theorem 3.6). Let $\mathscr{W}=\left\{\left(W_{i}, v_{i}\right)\right\}_{i=1}^{m}$ be a Parseval fusion frame for an $n$-dimension Hilbert space $\mathbb{F}^{n}$. Then the following are equivalent.

(i) The Parseval fusion frame $\mathscr{W}$ satisfies

$$
\begin{gathered}
e_{1}(\mathscr{W})=\min \left\{e_{1}\left(\left\{\left(\widetilde{W}_{i}, \widetilde{v}_{i}\right)\right\}_{i=1}^{m}\right):\right. \\
\left\{\left(\widetilde{W}_{i}, \widetilde{v}_{i}\right)\right\}_{i=1}^{m} \text { is a Parseval fusion } \\
\text { frame with } \operatorname{dim} \widetilde{W}_{i} \\
\left.=\operatorname{dim} W_{i}, \forall 1 \leq i \leq m\right\} .
\end{gathered}
$$

(ii) We have

$$
v_{i}^{2}=\frac{\operatorname{dim} \mathscr{H}}{m \cdot \operatorname{dim} W_{i}}, \quad \forall 1 \leq i \leq m .
$$

Moreover, let $f \in \mathscr{H}$ and $\tilde{f}$ be the reconstructed vector. Then we have the following error bound

$$
\|f-\widetilde{f}\| \leq \frac{\operatorname{dim} \mathscr{H}}{m \cdot \min \left\{\operatorname{dim} W_{i}: 1 \leq i \leq m\right\}} .
$$

The following proposition which follows from Proposition 7 and Theorem 13 describe the construction of one kind of optimal Parseval fusion frames for one packet erasure.

Proposition 14 (c.f. [19], Proposition 3.8). Let $\left\{e_{i}\right\}_{i=1}^{n}$ be an orthonormal basis for a Hilbert space $\mathbb{F}^{n}$, and let $k$ be a positive integer. Assume that $W_{j}$ is a subspace spanned by some elements in $\left\{e_{i}\right\}_{i=1}^{n}$ for $j=1,2, \ldots, m$, and $\operatorname{span}\left\{W_{j}\right\}_{j=1}^{m}=\mathscr{H}$. Let $h_{i}$ be number of subspaces $W_{j}$ that contain $e_{i}(1 \leq i \leq n)$. If all $\operatorname{dim} W_{j}$ are equal to $l$ and all $h_{i}$ are equal to $h$, then $\left\{\left(W_{j}, \sqrt{v_{j}}\right)\right\}_{j=1}^{m}$ is an optimal Parseval fusion frame for one packet erasure described in Theorem 13, where $v_{j}=n / m l$ for all $1 \leq j \leq m$.

The optimal Parseval fusion frame systems with local Parseval frames of prescribed numbers of frame vectors and prescribed dimensions of subspaces under the erasure of one local frame vector is presented in [18]. We find that constructing this kind of fusion frame systems can be reduced to constructing the conventional optimal Parseval frames with respect to one frame vector erasure under our method. Now let us first recall the related knowledge.

Let $\left(D_{1}, D_{2}, \ldots, D_{m}\right) \in \prod_{i=1}^{m} M\left(k_{i} \times k_{i}, \mathbb{F}\right)$ be a vector of matrices, where $D_{i_{0}}=\left(d_{i j}\right)_{k_{i_{0}} \times k_{i_{0}}}, d_{i j}=\delta_{i, j_{0}} \delta_{j, j_{0}}$ for some $i_{0} \in\{1,2, \ldots, m\}, j_{0} \in\left\{1,2, \ldots, k_{i_{0}}\right\}$, and other matrices are all zero-matrices, which simulate the erasure of vector $f_{i_{0} j_{0}}$. Denote the set of all these matrix vectors by $\mathscr{D}$. 
Definition 15. Let $\mathscr{W}=\left\{\left(W_{i}, v_{i},\left\{f_{i j}\right\}_{j=1}^{k_{i}}\right)\right\}_{i=1}^{m}$ be a Parseval fusion frame system with local Parseval frames. Let $\Theta_{\mathscr{W}}$ denote the analysis operator of the associated fusion frame, and $\Theta_{F_{i}}$ the analysis operator of the local frames for $1 \leq$ $i \leq m$. Then the associated 1-erasure of local frame vector reconstruction error is defined to be

$$
e_{1}^{*}(\mathscr{W})=\max \left\{\left\|\sum_{i=1}^{m} v_{i}^{2} \Theta_{F_{i}}^{*} D_{i} \Theta_{F_{i}}\right\|:\left(D_{1}, D_{2}, \ldots, D_{m}\right) \in \mathscr{D}\right\} .
$$

The following theorem characterizes the optimal Parseval fusion frame systems with subspaces of fixed dimensions and local Parseval frames having fixed numbers of frame vectors under one local frame vector erasure.

Theorem 16 (c.f. [18], Theorem 4.3). Let $\mathscr{W}=\left\{\left(W_{i}, v_{i}\right.\right.$, $\left.\left.\left\{f_{i j}\right\}_{j=1}^{k_{i}}\right)\right\}_{i=1}^{m}$ be a Parseval fusion frame system with local Parseval frames for an $n$-dimension Hilbert space $\mathbb{F}^{n}$. Then the following are equivalent:

(i) The Parseval fusion frame system satisfies $e_{1}^{*}(\mathscr{W})=$ $\min \left\{e_{1}^{*}\left(\left\{\left(\widetilde{W}_{i}, \widetilde{v}_{i},\left\{\widetilde{f}_{i j}\right\}_{j=1}^{k_{i}}\right)\right\}_{i=1}^{m}\right):\left\{\left(\widetilde{W}_{i}, \widetilde{v}_{i},\left\{\widetilde{f}_{i j}\right\}_{j=1}^{k_{i}}\right)\right\}_{i=1}^{m}\right.$ is a Parseval fusion frame system with local Parseval frames satisfying $\operatorname{dim} \widetilde{W}_{i}=\operatorname{dim} W_{i}$, for all $\left.1 \leq i \leq m\right\}$.

(ii) We have

$$
\left\|f_{i j}\right\|^{2}=\frac{\operatorname{dim} W_{i}}{k_{i}}, \quad \forall 1 \leq i \leq m, 1 \leq j \leq k_{i} .
$$

Moreover, let $f \in \mathscr{H}$ and $\tilde{f}$ be the reconstructed vector. Then we have the following error bound

$$
\|f-\widetilde{f}\| \leq \frac{\max \left\{\operatorname{dim} W_{i}: 1 \leq i \leq m\right\}}{\min \left\{k_{i}: 1 \leq i \leq m\right\}}\|f\| .
$$

The following proposition can be easily obtained by using the above theorem together with Proposition 2.1 of [9] and Theorem 8 . We omit its proof.

Proposition 17. Let $G_{i}=\left\{g_{i j}\right\}_{j=1}^{k_{i}}$ be the optimal Parseval frames with 1-erasure of $\mathbb{F}^{l_{i}}$ for $i=1,2, \ldots, m$, let $\left\{\left(W_{i}, v_{i},\right)\right\}_{i=1}^{m}$ be a Parseval fusion frame of $\mathbb{F}^{n}$ endowed with an orthonormal basis $\left\{e_{i j}\right\}_{j=1}^{l_{i}}$ for each subspace $W_{i}$. Set $L_{i}=\left(e_{i 1}, e_{i 2}, \ldots, e_{i l_{i}}\right)^{*}$, and $F_{i}=\left\{f_{i j}=L_{i}^{*} g_{i j}\right\}_{j=1}^{k_{i}}$ for all $1 \leq i \leq m$. Then $\mathscr{W}=$ $\left\{\left(W_{i}, v_{i},\left\{f_{i j}\right\}_{j=1}^{k_{i}}\right)\right\}_{i=1}^{m}$ is an optimal Parseval fusion frame system with local Parseval frames for one local frame vector erasure described in Theorem 16.

The above proposition provide a method for constructing the optimal Parseval fusion frame systems for one local frame vector erasure described in Theorem 16. First, we construct the optimal Parseval frames for 1-erasure in Hilbert space $\mathbb{F}^{l_{i}}$ for $i=1,2, \ldots, m$. Then, by using the algorithm presented by the above subsection we can derive the required optimal Parseval fusion frame system $\mathscr{W}=\left\{\left(W_{i}, v_{i},\left\{f_{i j}\right\}_{j=1}^{k_{i}}\right)\right\}_{i=1}^{m}$. Finally, we give a concrete example.
Example 18. Consider Hilbert space $\mathscr{H}=\mathbb{R}^{4}$, and let

$$
S=\left[\begin{array}{rrrr}
\frac{22}{3} & -\frac{2}{3} & -\frac{2}{3} & 0 \\
-\frac{2}{3} & \frac{22}{3} & -\frac{2}{3} & 0 \\
-\frac{2}{3} & -\frac{2}{3} & \frac{22}{3} & 0 \\
0 & 0 & 0 & 6
\end{array}\right]
$$

The eigenvalues of $S$ are $\lambda_{1}=\lambda_{2}=8, \lambda_{3}=\lambda_{4}=6$, and the corresponding orthonormal eigenvectors are given by

$$
\begin{gathered}
e_{1}=\left[\begin{array}{c}
-\frac{\sqrt{2}}{2} \\
\frac{\sqrt{2}}{2} \\
0 \\
0
\end{array}\right], \quad e_{2}=\left[\begin{array}{c}
-\frac{\sqrt{6}}{6} \\
-\frac{\sqrt{6}}{6} \\
\frac{2 \sqrt{6}}{6} \\
0
\end{array}\right], \\
e_{3}=\left[\begin{array}{c}
-\frac{\sqrt{3}}{6} \\
-\frac{\sqrt{3}}{6} \\
-\frac{\sqrt{3}}{6} \\
\frac{\sqrt{3}}{2}
\end{array}\right], \quad e_{4}=\left[\begin{array}{c}
\frac{1}{2} \\
\frac{1}{2} \\
\frac{1}{2} \\
\frac{1}{2}
\end{array}\right] .
\end{gathered}
$$

Let $W_{1}=\operatorname{span}\left\{e_{1}, e_{2}\right\}, W_{2}=\operatorname{span}\left\{e_{1}, e_{3}\right\}, W_{3}=\operatorname{span}\left\{e_{1}, e_{4}\right\}$, $W_{4}=\operatorname{span}\left\{e_{2}, e_{3}\right\}, W_{5}=\operatorname{span}\left\{e_{2}, e_{4}\right\}$, and $W_{6}=\operatorname{span}\left\{e_{3}, e_{4}\right\}$. According to the condition (7), we need positive solutions for the following equations:

$$
\begin{array}{ll}
v_{1}+v_{2}+v_{3}=8, & v_{1}+v_{4}+v_{5}=8 \\
v_{2}+v_{4}+v_{6}=6, & v_{3}+v_{5}+v_{6}=6 .
\end{array}
$$

These equations have infinite many positive solutions which can be expressed as

$$
\begin{gathered}
v_{1}=b+2, \quad v_{2}=a, \\
v_{3}=v_{4}=-a-b+6, \\
v_{5}=a, \quad v_{6}=b,
\end{gathered}
$$

where $a+b<2, a>0, b>0$. For example, we can take $a=b=2$, then we have $v_{1}=4, v_{2}=v_{3}=v_{4}=v_{5}=v_{6}=2$. Then we get a fusion frame $\left\{\left(W_{i}, v_{i}\right)\right\}_{i=1}^{4}$.

We can obtain a harmonic Parseval frame $G=\left\{g_{1}, g_{2}, g_{3}\right\}$ for $\mathbb{F}^{2}$ by using Example 4.1 in [8], where $g_{1}=(\sqrt{6} / 3,0)^{T}$, $g_{2}=(-\sqrt{6} / 6, \sqrt{2} / 2)^{T}$, and $g_{3}=(-\sqrt{6} / 6,-\sqrt{2} / 2)^{T}$. Since all vectors in $G$ have the same norm $\sqrt{6} / 3$, it is an 
optimal Parseval frame for 1-erasure by Proposition 2.1 of [9]. Set

$$
\begin{aligned}
& L_{1}=\left[\begin{array}{cccc}
-\frac{\sqrt{2}}{2} & \frac{\sqrt{2}}{2} & 0 & 0 \\
-\frac{\sqrt{6}}{6} & -\frac{\sqrt{6}}{6} & \frac{2 \sqrt{6}}{6} & 0
\end{array}\right] \text {, } \\
& L_{2}=\left[\begin{array}{cccc}
-\frac{\sqrt{2}}{2} & \frac{\sqrt{2}}{2} & 0 & 0 \\
-\frac{\sqrt{3}}{6} & -\frac{\sqrt{3}}{6} & -\frac{\sqrt{3}}{6} & \frac{\sqrt{3}}{2}
\end{array}\right] \text {, } \\
& L_{3}=\left[\begin{array}{cccc}
-\frac{\sqrt{2}}{2} & \frac{\sqrt{2}}{2} & 0 & 0 \\
\frac{1}{2} & \frac{1}{2} & \frac{1}{2} & \frac{1}{2}
\end{array}\right], \\
& L_{4}=\left[\begin{array}{cccc}
-\frac{\sqrt{6}}{6} & -\frac{\sqrt{6}}{6} & \frac{2 \sqrt{6}}{6} & 0 \\
-\frac{\sqrt{3}}{6} & -\frac{\sqrt{3}}{6} & -\frac{\sqrt{3}}{6} & \frac{\sqrt{3}}{2}
\end{array}\right] \text {, } \\
& L_{5}=\left[\begin{array}{cccc}
-\frac{\sqrt{6}}{6} & -\frac{\sqrt{6}}{6} & \frac{2 \sqrt{6}}{6} & 0 \\
\frac{1}{2} & \frac{1}{2} & \frac{1}{2} & \frac{1}{2}
\end{array}\right] \text {, } \\
& L_{6}=\left[\begin{array}{cccc}
-\frac{\sqrt{3}}{6} & -\frac{\sqrt{3}}{6} & -\frac{\sqrt{3}}{6} & \frac{\sqrt{3}}{2} \\
\frac{1}{2} & \frac{1}{2} & \frac{1}{2} & \frac{1}{2}
\end{array}\right] .
\end{aligned}
$$

We compute $\left\{f_{i j}\right\}_{i=1, j=1}^{6,3}$ as follows:

$$
\begin{gathered}
f_{11}=L_{1}^{*} g_{1}=\left(-\frac{\sqrt{3}}{3}, \frac{\sqrt{3}}{3}, 0,0\right)^{T}, \\
f_{12}=L_{1}^{*} g_{2}=\left(0,-\frac{\sqrt{3}}{3}, \frac{\sqrt{3}}{3}, 0\right)^{T}, \\
f_{13}=L_{1}^{*} g_{3}=\left(\frac{\sqrt{3}}{3}, 0,-\frac{\sqrt{3}}{3}, 0\right)^{T}, \\
f_{21}=L_{2}^{*} g_{1}=\left(-\frac{\sqrt{3}}{3}, \frac{\sqrt{3}}{3}, 0,0\right)^{T}, \\
f_{22}=L_{2}^{*} g_{2}=\left(\frac{2 \sqrt{3}-\sqrt{6}}{12},-\frac{2 \sqrt{3}+\sqrt{6}}{12},-\frac{\sqrt{6}}{12}, \frac{\sqrt{6}}{4}\right)^{T}, \\
f_{23}=L_{2}^{*} g_{3}=\left(\frac{2 \sqrt{3}+\sqrt{6}}{12},-\frac{2 \sqrt{3}-\sqrt{6}}{12}, \frac{\sqrt{6}}{12},-\frac{\sqrt{6}}{4}\right)^{T},
\end{gathered}
$$

$$
\begin{gathered}
f_{31}=L_{3}^{*} g_{1}=\left(-\frac{\sqrt{3}}{3}, \frac{\sqrt{3}}{3}, 0,0\right)^{T}, \\
f_{32}=L_{3}^{*} g_{2}=\left(\frac{2 \sqrt{3}+3 \sqrt{2}}{12},-\frac{2 \sqrt{3}-3 \sqrt{2}}{12}, \frac{\sqrt{2}}{4}, \frac{\sqrt{2}}{4}\right)^{T}, \\
f_{33}=L_{3}^{*} g_{3}=\left(\frac{2 \sqrt{3}-3 \sqrt{2}}{12},-\frac{2 \sqrt{3}+3 \sqrt{2}}{12},-\frac{\sqrt{2}}{4},-\frac{\sqrt{2}}{4}\right)^{T}, \\
f_{41}=L_{4}^{*} g_{1}=\left(-\frac{1}{3},-\frac{1}{3}, \frac{2}{3}, 0\right)^{T}, \\
f_{42}=L_{4}^{*} g_{2}=\left(\frac{2-\sqrt{6}}{12}, \frac{2-\sqrt{6}}{12},-\frac{4+\sqrt{6}}{12}, \frac{\sqrt{6}}{4}\right)^{T}, \\
f_{51}=\left(\frac{2+\sqrt{6}}{12}, \frac{2+\sqrt{6}}{12},-\frac{4-\sqrt{6}}{12},-\frac{\sqrt{6}}{4}\right)^{T}, \\
f_{63} g_{1}=\left(-\frac{1}{3},-\frac{1}{3}, \frac{2}{3}, 0\right)^{T}, \\
f_{52}=L_{5}^{*} g_{2}=\left(\frac{2+3 \sqrt{2}}{12}, \frac{2+3 \sqrt{2}}{12},-\frac{4-3 \sqrt{2}}{12}, \frac{\sqrt{2}}{4}\right)^{T}, \\
\left.f_{61}=\left(-\frac{\sqrt{2}}{6},-\frac{\sqrt{2}}{6},-\frac{\sqrt{2}}{6},-\frac{\sqrt{2}}{2}\right)^{*} g_{1}=\left(-\frac{\sqrt{2}}{6}, \frac{\sqrt{2}}{3}, 0\right)^{T},-\frac{\sqrt{2}}{6}, \frac{\sqrt{2}}{2}\right)^{T}, \\
f_{53}=L_{5}^{*} g_{3}=\left(\frac{2-3 \sqrt{2}}{12}, \frac{2-3 \sqrt{2}}{12},-\frac{4+3 \sqrt{2}}{12},-\frac{\sqrt{2}}{4}\right)^{T},
\end{gathered}
$$

Then $\mathscr{W}=\left\{\left(W_{j}, v_{j},\left\{f_{i j}\right\}_{j=1}^{3}\right)\right\}_{j=1}^{6}$ is an optimal Parseval fusion frame system with local Parseval frames under one local frame vector erasure in the sense of Theorem 16 by Proposition 17.

The original gray image of windmill is shown in Figure 1. We encode the data of the image by using the local frames of the Parseval fusion frame system given by this example. Then we decode the coded data, where first element of every local vector is deleted by using the Parseval fusion frame of this example. The reconstructed image is shown in Figure 2. One can observe the reconstruction effect by comparing the two figures.

\section{Conclusion}

We studied the method for constructing a fusion frame system in a finite-dimensional Hilbert space $\mathbb{F}^{n}$ according to its fusion frame operator matrix in this paper. The corresponding algorithm was given. Then we obtained the 


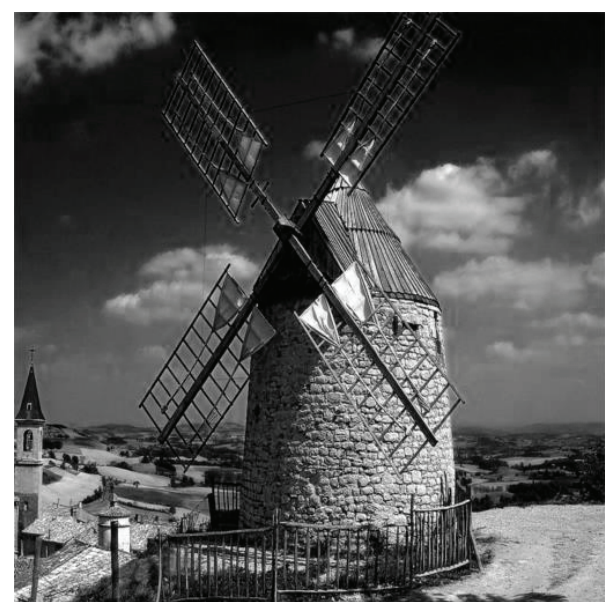

FIGURE 1: The original gray image of windmill.

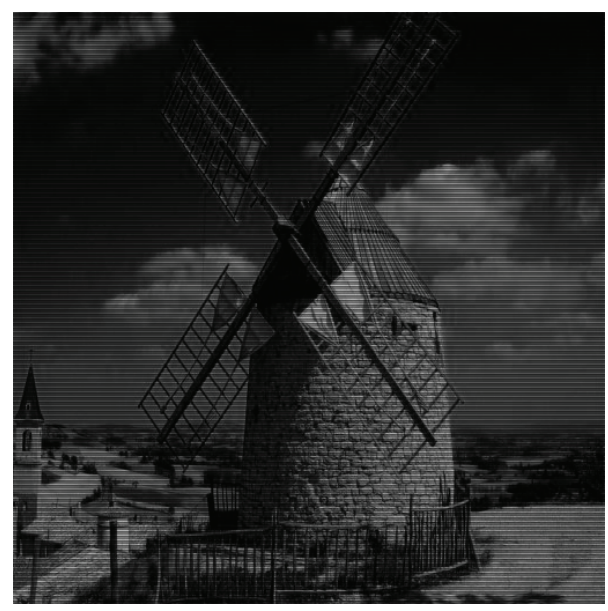

FIgURE 2: The reconstructed gray image of windmill. The data of the original image is encoded by the local frames of the Parseval fusion frame system computed by Example 18. The first coefficient of every local vector is deleted. The remained data is decoded by the Parseval fusion frame of Example 18.

matrix representations of its local frame operators and inverse frame operators and researched the related characteristics of these fusion frame systems. We provided methods to get the optimal fusion frame systems for erasures in some special sense in signal transmission. Finally, we constructed a fusion frame system as an example by our method and successfully applied it in image coding.

\section{Conflict of Interests}

The authors declare that there is no conflict of interests regarding the publication of this paper.

\section{Acknowledgments}

The authors wish to thank the anonymous reviewers for their valuable comments and suggestions that have improved the presentation of this paper. This work was supported by the National Natural Science Foundation of China (11271001 and 61370147), 973 Program (2013CB329404), and Sichuan Province Science and Technology Research Project (12ZC1802).

\section{References}

[1] I. Cidon, H. Kodesh, and M. Sidi, "Erasure, capture, and random power level selection in multiple-access systems," IEEE Transactions on Communications, vol. 36, no. 3, pp. 263-271, 1988.

[2] A. F. Dana, R. Gowaikar, R. Palanki, B. Hassibi, and M. Effros, "Capacity of wireless erasure networks," IEEE Transactions on Information Theory, vol. 52, no. 3, pp. 789-804, 2006.

[3] D. Han, K. Kornelson, D. Larson, and E. Weber, Frames for Undergraduates, vol. 40 of Mathematical Library Book Series, American Mathematical Society, Providence, RI, USA, 2007.

[4] E. J. Candès and D. L. Donoho, "New tight frames of curvelets and optimal representations of objects with piecewise $C^{2}$ singularities," Communications on Pure and Applied Mathematics, vol. 57, no. 2, pp. 219-266, 2004.

[5] B. G. Bodmann and V. I. Paulsen, "Frame paths and error bounds for sigma-delta quantization," Applied and Computational Harmonic Analysis, vol. 22, no. 2, pp. 176-197, 2007.

[6] C. H. Bennett, D. P. DiVincenzo, and J. A. Smolin, "Capacities of quantum erasure channels," Physical Review Letters, vol. 78, no. 16, pp. 3217-3220, 1997.

[7] B. G. Bodmann and V. I. Paulsen, "Frames, graphs and erasures," Linear Algebra and its Applications, vol. 404, pp. 118-146, 2005.

[8] V. K. Goyal, J. Kovačević, and J. A. Kelner, "Quantized frame expansions with erasures," Applied and Computational Harmonic Analysis, vol. 10, no. 3, pp. 203-233, 2001.

[9] R. B. Holmes and V. I. Paulsen, "Optimal frames for erasures," Linear Algebra and its Applications, vol. 377, pp. 31-51, 2004.

[10] J. Leng and D. Han, “Optimal dual frames for erasures II," Linear Algebra and its Applications, vol. 435, no. 6, pp. 1464-1472, 2011.

[11] J. S. Leng, D. Han, and T. Huang, "Optimal dual frames for communication coding with probabilistic erasures," IEEE Transactions on Signal Processing, vol. 59, no. 11, pp. 5380-5389, 2011.

[12] J. S. Leng, D. Han, and T. Huang, "Probability modelled optimal frames for erasures," Linear Algebra and its Applications, vol. 438, no. 11, pp. 4222-4236, 2013.

[13] A. Albanese, J. Blömer, J. Edmonds, M. Luby, and M. Sudan, "Priority encoding transmission," IEEE Transactions on Information Theory, vol. 42, no. 6, part 1, pp. 1737-1744, 1996.

[14] P. G. Casazza and G. Kutyniok, "Frames of subspaces," in Wavelets, Frames, and Operator Theory, vol. 345 of Contemporary Mathematics Series, pp. 87-113, American Mathematical Society, Providence, RI, USA, 2004.

[15] P. G. Casazza, G. Kutyniok, and S. Li, "Fusion frames and distributed processing," Applied and Computational Harmonic Analysis, vol. 25, no. 1, pp. 114-132, 2008.

[16] P. G. Casazza and M. Fickus, "Minimizing fusion frame potential," Acta Applicandae Mathematicae, vol. 107, no. 1-3, pp. 7-24, 2009.

[17] P. G. Casazza, M. Fickus, D. G. Mixon, Y. Wang, and Z. Zhou, "Constructing tight fusion frames," Applied and Computational Harmonic Analysis, vol. 30, no. 2, pp. 175-187, 2011. 
[18] P. G. Casazza and G. Kutyniok, "Robustness of fusion frames under erasures of subspaces and of local frame vectors," in Radon Transforms, Geometry, and Wavelets, vol. 464 of Contemporary Mathematics Series, pp. 149-160, American Mathematical Society, Providence, RI, 2008.

[19] J. S. Leng and D. Han, "Orthogonal projection decomposition of matrices and construction of fusion frames," Advances in Computational Mathematics, vol. 38, no. 2, pp. 369-381, 2013.

[20] P. G. Massey, M. A. Ruiz, and D. Stojanoff, "The structure of minimizers of the frame potential on fusion frames," The Journal of Fourier Analysis and Applications, vol. 16, no. 4, pp. 514-543, 2010.

[21] P. G. Casazza, G. Kutyniok, S. Li, and C. J. Rozell, "Modeling sensor networks with fusion frames," in Wavelets XII, vol. 6701 of Proceedings of SPIE, San Diego, Calif, USA, August 2007.

[22] B. G. Bodmann, "Optimal linear transmission by lossinsensitive packet encoding," Applied and Computational Harmonic Analysis, vol. 22, no. 3, pp. 274-285, 2007.

[23] B. G. Bodmann and G. Kutyniok, "Erasure-proof transmissions: fusion frames meet coding theory," in Wavelets XIII, vol. 7446 of Proceedings of SPIE, San Diego, Calif, USA, August 2009.

[24] B. G. Bodmann, D. W. Kribs, and V. I. Paulsen, "Decoherenceinsensitive quantum communication by optimal $C^{a s t}$ encoding," IEEE Transactions on Information Theory, vol. 53, no. 12, pp. 4738-4749, 2007.

[25] J. S. Leng, Q. X. Guo, and T. Z. Huang, "The duals of fusion frames for experimental data transmission coding of high energy physics," Advances in High Energy Physics, vol. 2013, Article ID 837129, 9 pages, 2013.

[26] C. Cattani and A. Ciancio, "Separable transition density in the hybrid model for tumor-immune system competition," Computational and Mathematical Methods in Medicine, vol. 2012, Article ID 610124, 6 pages, 2012.

[27] C. Cattani, A. Ciancio, and B. Lods, "On a mathematical model of immune competition," Applied Mathematics Letters, vol. 19, no. 7, pp. 678-683, 2006.

[28] C. Cattani and L. M. S. Ruiz, "Discrete differential operators in multidimensional Haar wavelet spaces," International Journal of Mathematics and Mathematical Sciences, no. 41-44, pp. 23472355, 2004. 


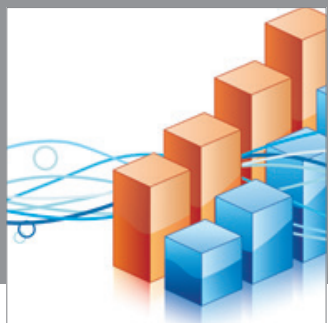

Advances in

Operations Research

mansans

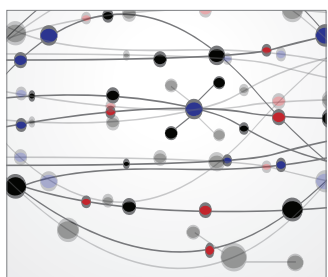

The Scientific World Journal
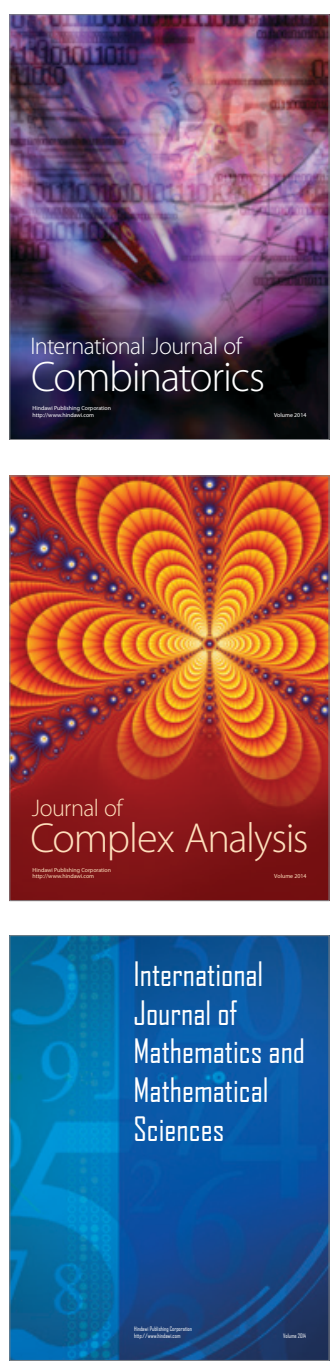
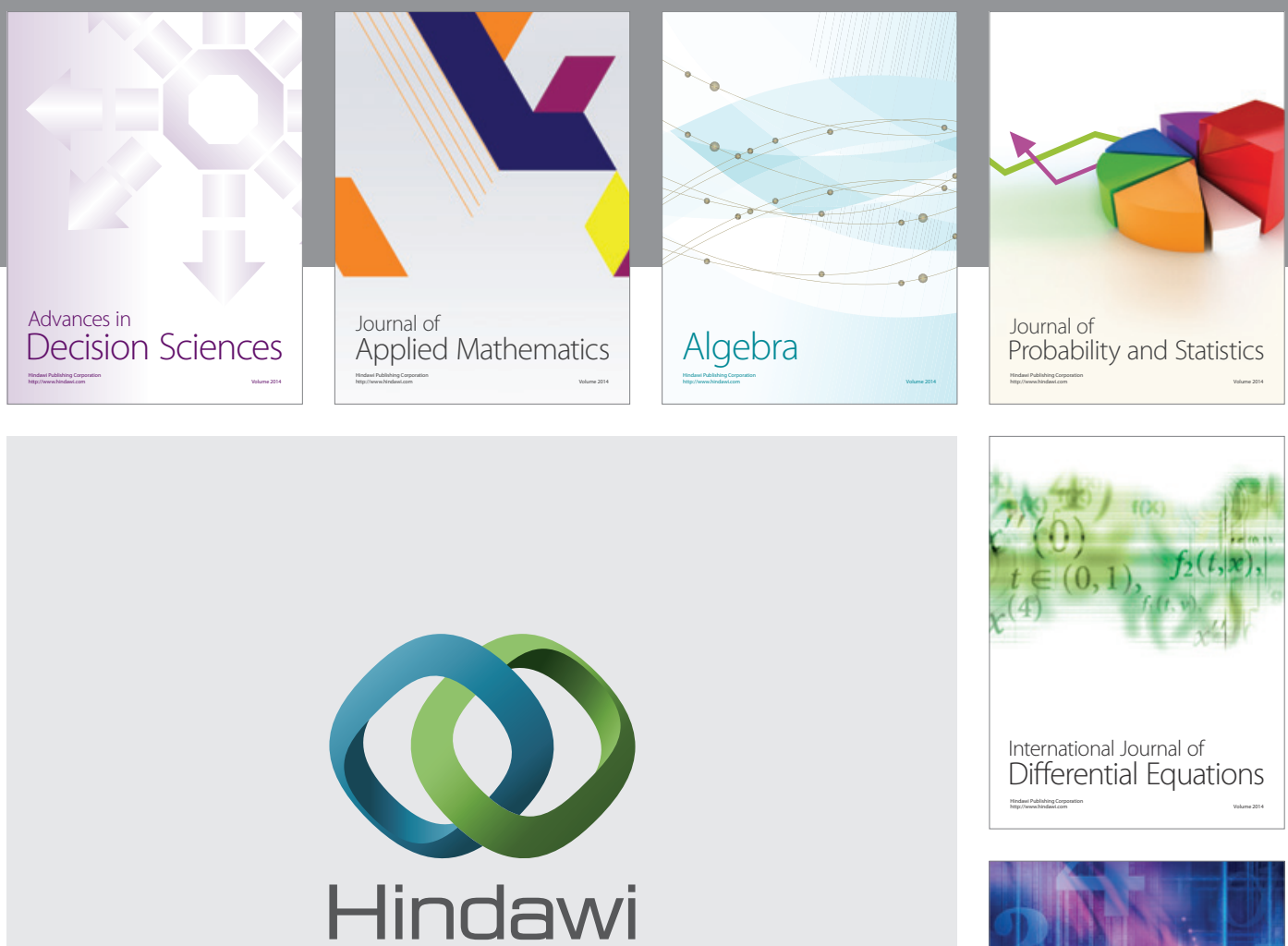

Submit your manuscripts at http://www.hindawi.com
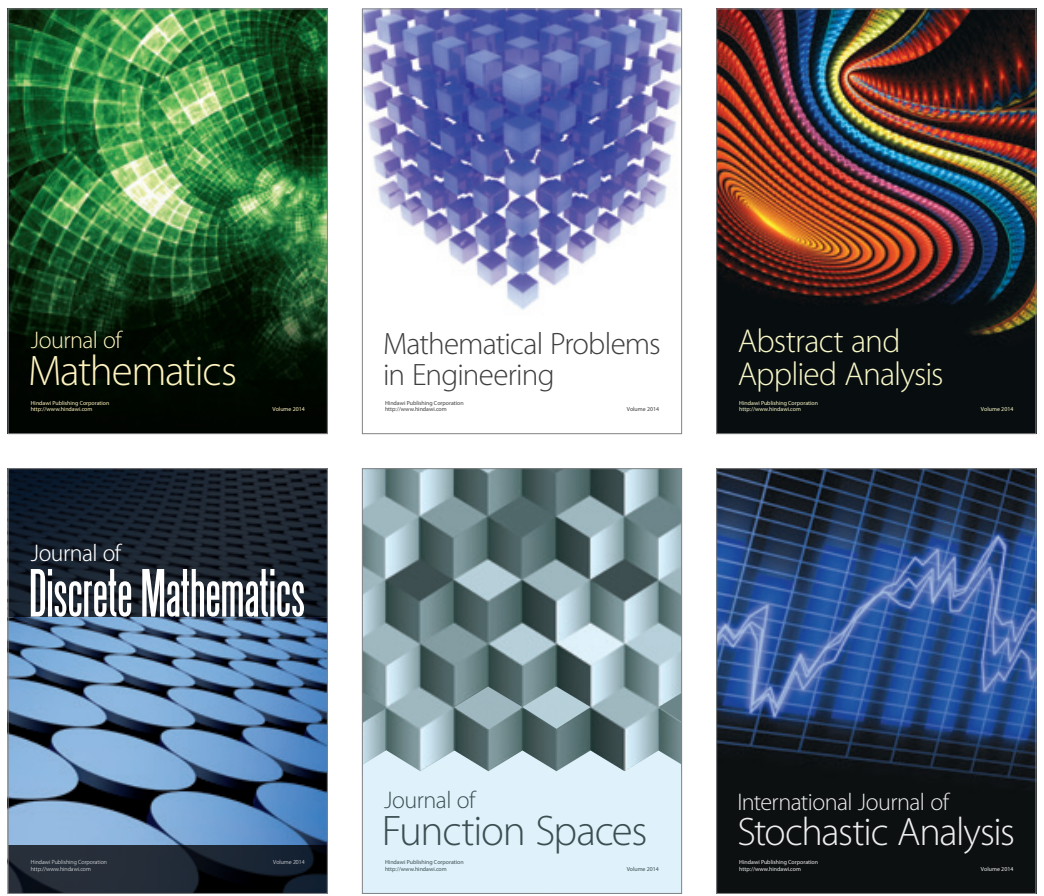

Journal of

Function Spaces

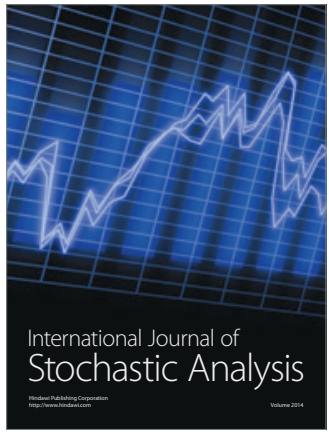

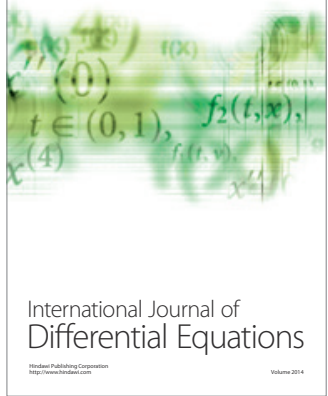
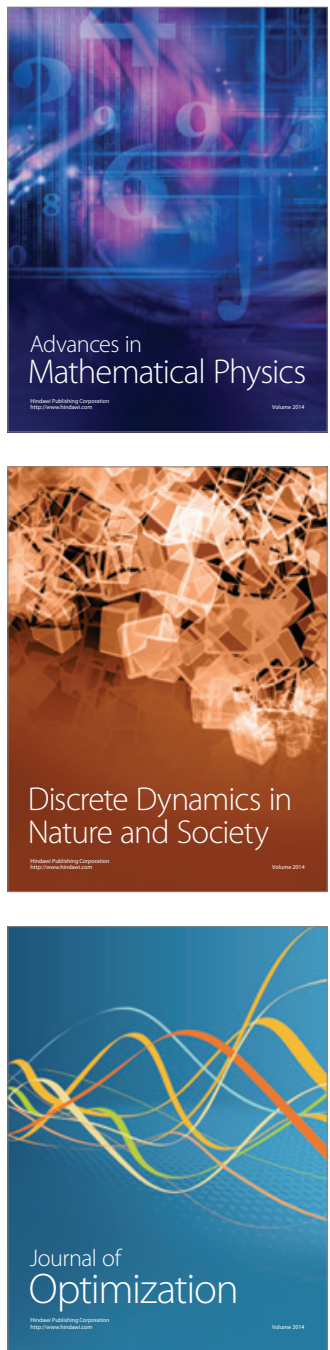